\title{
Efeitos da ingestão dos aminoácidos de cadeia ramificada na fadiga central
}

\author{
Paulo Armada-da-Silva \\ Francisco Alves
}

https://doi.org/10.5628/rpcd.05.01.102

\author{
Universidade Técnica de Lisboa \\ Faculdade de Motricidade Humana \\ Portugal
}

\begin{abstract}
RESUMO
A descoberta de alterações na actividade e concentração intracefálica de alguns neurotransmissores, nomeadamente a serotonina (5-hidroxitriptamina, 5-HT), durante e após o exercício intenso de longa duração, colocou a questão da relação entre o conhecido efeito depressor destes compostos e o controlo metabólico em esforço de dominante aeróbia. A perturbação do funcionamento dos sistemas monoaminérgicos encefálicos induzida pelo exercício pode estar na base do impedimento à continuação da activação central do sistema neuromuscular em situações próximas do limite, tão habituais no atleta de fundo quando em competição. Ora, sabe-se que a taxa de síntese da 5-HT é sensível à concentração plasmática do triptofano (TRP) livre, dos aminoácidos de cadeia ramificada (AACR) e dos ácidos gordos, o que permitirá a manipulação da função serotoninérgica indirectamente através da suplementação de AACR e de hidratos de carbono. A exploração da intervenção dietética ganhou importância devido à probabilidade da suplementação em AACR induzir uma redução da taxa de passagem dos percursores da 5-HT pela barreira hemato-encefálica. É nosso objectivo neste trabalho discutir a eficácia da intervenção dietética sobre os mecanismos subjacentes ao fenómeno da fadiga central a partir da apreciação do quadro actual de conhecimentos sobre estes complexos mecanismos de regulação metabólica e neuro-humoral.
\end{abstract}

Palavras-chave: fadiga central, aminoácidos de cadeia ramificada, serotonina.

\begin{abstract}
The role of branched chain amino acids supplementation on central fatigue.

Evidence of changes in the concentration of certain neurotransmitters in the brain during and after long distance high-intensity exercise, especially serotonin (5-hydroxytryptamine, 5-HT), raised the question of the relationship between the well known depressor effect of these compounds and the metabolic and neuro-hormonal regulation present in the acute response to this type of physical exertion. Functional disturbance of the brain monoaminergic systems induced may be the reason for the inability to maintain central activation of muscle in near the limit situations as happens so often to long distance athletes in competition. Since it is well known that the rate of synthesis of 5-HT is sensitive to the plasma content in free triptophan (TRP), branched-chain amino acids (BCAA) and free fatty acids, it has been postulated that an increase in free fatty acids during and after endurance exercise mediates an increment of free TRP which, in combination with a decline of circulating BCAA, facilitates the entry of TRP into the brain, there by increasing cerebral serotonergic tone. This way, supplementation with $B C A A s$ and/or carbohydrates during exercise becomes an interesting procedure to explore, since it may reduce the rate of penetration of 5 HT precursors through the blood-brain barrier. The aim of this review is to discuss the efficacy of nutritional strategies on the mechanisms underlying central fatigue from present knowledge on the complex metabolic and neurohumoral regulation mechanisms.
\end{abstract}

Key Words: central fatigue, branched-chain amino acids, serotonin. 


\section{INTRODUÇÃO}

O termo fadiga designa a redução da capacidade do músculo em produzir força e desenvolver potência. Para além das alterações objectivas, a fadiga muscular ocorre também associada a manifestações subjectivas, como o aumento da percepção de esforço dissociado de variações da intensidade de exercício. A fadiga durante o exercício físico é um fenómeno complexo cujas causas parecem depender do tipo de esforço, existindo algum consenso actualmente na distinção entre esforços curtos e intensos, por um lado, e esforços prolongados, por outro. Em esforços curtos e de intensidade máxima ou supra-máxima, o decréscimo da produção de trabalho muscular está grandemente associado a factores intramusculares, tais como redução do pH muscular, aumento da concentração do fosfato inorgânico no interior da fibra muscular, ou falha na libertação sarcoplásmica de $\mathrm{Ca}^{2+}$ [43]. No caso dos esforços prolongados, porém, as alterações contrácteis do músculo esquelético parecem não ser acentuadas $[42,44,56]$, o que sugere que outros factores, designadamente factores metabólicos tais como a redução acentuada de substratos para o metabolismo energético ou a ocorrência de hipoglicémia, poderão estar na origem do surgimento da fadiga muscular $[15,34]$.

Na década de sessenta do passado século, o uso da biópsia muscular para estudo do metabolismo muscular durante o exercício veio confirmar o papel essencial desempenhado pelo glicogénio muscular na capacidade de realização de exercício $[15,16,51]$. Num estudo muito influente, Bergström et al. [15] demonstraram com clareza o aumento da capacidade de realização de exercício de intensidade moderada em virtude de uma dieta rica em hidratos de carbono, quando comparada a uma dieta mais rica em gorduras, efeito que foi explicado pelo aumento da quantidade de glicogénio em reserva no músculo esquelético. Os resultados obtidos neste estudo, e que vieram a ser comprovados por investigações posteriores, colocaram a depleção do glicogénio muscular como a mais provável causa de fadiga em esforços longos $(>2 \mathrm{~h}$ ) e de intensidade moderada (60-80\% $\mathrm{VO}_{2 \text { máx }}$ [34]. A diminuição da taxa de degradação e utilização do glicogénio muscular passou também a servir de explicação para o efeito positivo na resistência à fadiga da ingestão de hidratos de carbono antes e durante o exercício [33], assim como para a acção ergogénica de algumas substâncias, em particular a cafeína [32].

Contudo, a relação entre fadiga em esforços de longa duração e a utilização das reservas de glicogénio muscular não é inequívoca [42]. Mais recentemente foi proposta uma explicação alternativa, que enfatiza o papel do sistema nervoso central (SNC) como causa de fadiga em esforços de longa duração. De acordo com a designada hipótese da fadiga central de Newsholme e colaboradores [70,71], a fadiga é o resultado do aumento da libertação cerebral de serotonina (5-hidroxitriptamina, 5-HT), que tem como causa factores metabólicos que levam ao aumento da incorporação cerebral do seu precursor, o aminoácido essencial triptofano (TRP). De acordo com esta hipótese, a actividade serotoninérgica cerebral é afectada pela duração do exercício, daí resultando dificuldade em manter a activação das áreas motoras cerebrais e a excitação muscular, às quais se associa também, directa ou indirectamente, o aumento da percepção subjectiva do esforço.

Várias abordagens experimentais têm sido seguidas na tentativa de demonstrar o papel da 5-HT na génese da fadiga durante exercício prolongado. Em humanos, uma boa parte da investigação tem recorrido à ingestão de aminoácidos de cadeia ramificada (AACR, leucina, valina, isoleucina), na tentativa de compensar as modificações metabólicas originadas pelo exercício e causadoras do aumento da quantidade de 5-HT cerebral e, deste modo, comprovar a associação entre 5-HT e fadiga [80, 81]. No âmbito desportivo, esta hipótese sugere que a ingestão de AACR antes e durante o exercício poderá beneficiar o desempenho competitivo em provas longas. Interessa assim conhecer qual o fundamento científico para a pressuposta eficácia desta estratégia ergogénica nutritiva e lícita. Esta curta revisão tem com objectivo sintetizar os principais resultados obtidos na investigação do papel desempenhado pelas vias serotoninérgicas na indução da fadiga em esforços prolongados que tem vindo a ser realizada nos últimos anos. Feita esta síntese, procurar-se-á avaliar qual a legitimidade, de um ponto de vista da evidência científica, no aconselhar a ingestão de AACR previamente ao exercício como forma de melhorar o desempenho desportivo. 


\section{AUMENTO DA SEROTONINA CEREBRAL COMO CAUSA DE FADIGA}

Nos últimos anos, o interesse sobre o papel desempenhado pelas vias monoaminérgicas, em particular as vias da 5-HT, na génese da fadiga em exercícios prolongados, tem vindo a crescer. A 5-HT, um dos principais neurotransmissores do sistema nervoso central, é fundamental para o normal funcionamento cerebral, participando na regulação de um conjunto vasto de funções biológicas através de uma acção de equilibração da neurotransmissão e da neuromodulação (ver Azmitia [7], e Struder e Weicker [80, 81]). O papel regulador da 5-HT sobre a generalidade das funções cerebrais, sobretudo no que respeita à regulação do ciclo sono-vigília, à modulação da dor e à regulação da actividade motora [53], torna plausível um papel directo nos factores centrais de fadiga $[70,71]$. A taxa de síntese de 5-HT é regulada pela actividade da enzima triptofano hidroxilase que, ao contrário das enzimas de síntese das outras monoaminas, não se encontra em saturação, de modo que a taxa de síntese da 5-HT varia de acordo com a concentração plasmática de TRP [93]. O teor do TRP cerebral depende da taxa com que este aminoácido atravessa o endotélio dos vasos cerebrais por intermédio do sistema de transporte dos aminoácidos neutros, sendo assim variável de acordo com a concentração da fracção livre do TRP (TRPlivre) e da concentração plasmática dos aminoácidos que partilham o mesmo sistema de transporte de membrana [57]. A proporção do TRP livre face ao TRP plasmático total (TRP total) depende, por seu lado, da concentração plasmática de ácidos gordos. Os ácidos gordos no plasma unem-se em parte à albumina, deslocando o TRP, fazendo aumentar a quantidade de TRP livre [35, 40]. Durante o exercício, a razão TRP livre/TRP total eleva-se em virtude do aumento progressivo da quantidade de ácidos gordos não esterificados em circulação. Os valores observados por Blomstrand et al. [17] em fundistas após corrida uma maratona traçaram, a este respeito, um quadro claro das alterações destes parâmetros: a concentração de ácidos gordos quadriplicou, a quantidade de TRP livre cresceu duas vezes e meia e a proporção do TRP livre face ao TRP total aumentou de $16 \%$ para $50 \%$. Entre os aminoácidos que partilham o transportador de membrana com o TRP incluem-se os AACR.
Assim, a razão TRP/AACR é outro potencial determinante da acumulação cerebral de TRP e, consequentemente, da síntese de 5-HT. Durante o exercício prolongado, o teor plasmático dos AACR tende a diminuir pelo aumento da taxa de entrada no músculo esquelético, criando-se, assim, condições de ocorrência da subida do valor do quociente TRP/AACR e do aumento da taxa de transporte do TRP através da barreira hemato-encefálica [2]. Dado que factores metabólicos sistémicos, como as concentrações plasmáticas de TRP, de outros aminoácidos e de ácidos gordos, alteram a síntese da 5HT, é possível modificar a actividade serotoninérgica cerebral pela ingestão de precursores da 5-HT, designadamente do TRP, ou de outros aminoácidos, particulamente os AACR [18, 20, 22, 25, 83].

Diversos estudos mostram que variações do aporte alimentar de TRP [5, 38], ou do teor plasmático deste aminoácido [30], afectam a actividade serotoninérgica cerebral. No caso do exercício prolongado, porém, os dados são escassos. van Hall e colaboradores [83] verificaram que a ingestão de TRP em quantidade suficiente para aumentar entre 7 a 20 vezes a sua concentração cerebral não afectou a capacidade de exercício durante um teste em cicloergómetro, a uma intensidade de $70-75 \%$ do pico de potência e numa amostra constituída por indivíduos treinados. Estudos em animais, porém, indicam que a concentração plasmática de TRP pode condicionar o desempenho físico. Num estudo realizado em cavalos, verificou-se redução significativa na resistência dos animais ao esforço após ingestão de TRP, mesmo quando associada à ingestão de glucose [41]. Por outro lado, um estudo realizado em ratos albuminémicos, os quais apresentam uma elevação do teor de TRP livre, parece validar a hipótese da fadiga central [91, 92]. Nestes animais, a inibição do transportador do TRP aumentou substancialmente a resistência à fadiga, efeito que esteve associado à diminuição da penetração do TRP e do 5-hidroxitriptofano (5-HTP) nas terminações serotoninérgicas do corpo estriado [92]. No homem existem algumas evidências indirectas indicadoras de existência de relação entre as alterações metabólicas referidas e o aumento da fatigabilidade que se ajustam ao modelo da fadiga central. Por exemplo, valores aumentados de concentração plasmática de TRP e da razão TRP/AACR são 
usuais em pacientes com o síndrome de fadiga crónica [26], assim como em estados pós-operatórios [91], o que sugere o envolvimento da 5-HT nos sintomas de fadiga que acompanham estas situações. A acção do TRP sobre a síntese e libertação de 5-HT parece depender do estado metabólico, com aumento do seu efeito após jejum prolongado [30], provavelmente devido à maior actividade lipolítica e/ou à acção das catecolaminas no transporte do TRP para o interior do sistema nervoso central [29]. Através de microdiálise, Meeusen et al. [66] verificaram um aumento da libertação de 5-HT na região do hipocampo (terminais serotoninérgicos) em ratos em privação alimentar e após a ingestão de TRP. Para além deste efeito na libertação basal de 5-HT, o aporte alimentar de TRP potenciou ainda a libertação daquele neurotransmissor durante o exercício [66].

Algumas das contradições encontradas entre estudos, no que respeita ao efeito da ingestão do TRP na actividade das vias da 5-HT cerebrais e na fadiga ao exercício, poderão ser devidas ao uso de diferentes protocolos experimentais, com variação no número de horas de jejum ou no controlo da dieta nos dias anteriores aos ensaios experimentais.

Acrescenta-se a este conjunto de limitações experimentais o facto da interpretação dos resultados obtidos com a ingestão de TRP dever ter em consideração a inexistência de relação directa entre a taxa de síntese de 5-HT e a taxa da sua libertação sináptica, dada a presença de autoreceptores serotoninérgicos no soma neuronal [14]. Por outro lado, há alguma indicação da possibilidade de alteração pelo exercício do controlo exercido pelo TRP sobre a síntese de 5-HT [28], possivelmente por inibição da actividade da triptofano hidroxilase [30], pelo que a relação entre o teor de TRP plasmático e a taxa de síntese de 5-HT, existente em repouso, pode não ser válida no caso do exercício. É possível, também, que a activação da enzima monoamina oxidase acompanhe o aumento da síntese de 5-HT estimulada pela maior concentração intra-neuronal de TRP, daí resultando aumento da taxa de degradação da 5-HT [63]. Em resumo, não é possível concluir sobre qual o papel da 5-HT na fadiga ao exercício com base em estudos de ingestão de TRP, dado a incerteza quanto ao efeito da maior quantidade de TRP cerebral na libertação de 5-HT durante o exercício.
Outro dos métodos para investigar o papel desempenhado pela 5-HT na fadiga durante exercício físico de longa duração consiste na utilização de fármacos com acção na actividade da 5-HT. Os resultados deste tipo de investigação são igualmente inconclusivos mas, pelo menos em modelos animais, sugerem que o aumento da quantidade cerebral de 5-HT induz fadiga [4, 8-10]. Em roedores, a fadiga deu-se de modo mais rápido após a administração de quipazina dimaleato, um agonista genérico da 5-HT, e de m-clorofenil-piperazina, um agonista dos receptores $5-\mathrm{HT}_{1 \mathrm{C}}$, tendo o efeito negativo da quipazina dimaleato sido abolido por inibição dos receptores serotoninérgicos $5-\mathrm{HT}_{1 \mathrm{C}}$ e $5-\mathrm{HT}_{2}$ [9]. Neste estudo foi ainda observada uma redução durante o exercício das concentrações cerebrais de dopamina e do seu catabolito, o ácido 3,4-dihidroxifenilacético, sugerindo que a relação entre 5-HT e fadiga possa ser devida à interacção dos dois neurotransmissores. Ao contrário do verificado para os receptores 5$\mathrm{HT}_{1 \mathrm{C}}$ e $5-\mathrm{HT}_{2}$, a activação dos receptores $5-\mathrm{HT}_{1 \mathrm{~A}}$ pelo agonista 8-OHDPAT [8-hidroxi-2-(di-n-propilamina) tetralina] não produziu alteração no comportamento motor dos ratos, ou no nível de fadiga durante corrida em passadeira rolante [52]. Porém, estudos mais recentes indicam que a estimulação farmacológica dos receptores $5-\mathrm{HT}_{1 \mathrm{~A}}$ afecta a resistência do rato à fadiga, mas com efeitos contrários de acordo com a dose utilizada [4]. A administração de 8-OHDPAT em doses baixas $(0.1 \mathrm{mg} / \mathrm{kg})$ parece aumentar a capacidade de corrida em tapete rolante no rato, enquanto doses mais elevadas do fármaco (0.2-0.8 $\mathrm{mg} / \mathrm{kg})$ produzem efeito contrário [4]. No homem, a administração de diferentes tipos de fármacos com acção nas vias da 5-HT tem produzido efeitos ambivalentes sobre o desempenho físico. Num estudo envolvendo exercício em cicloergómetro $\left(\sim 80 \% \mathrm{VO}_{2 \text { máx }}\right)$ até ao ponto de exaustão, a administração de um agonista parcial dos receptores $5-\mathrm{HT}_{1 \mathrm{~A}}$, a buspirona, reduziu em cerca de dois terços o tempo de exercício e elevou a percepção subjectiva de esforço ao longo do ensaio [64]. Contudo, a buspirona é um antagonista parcial dos receptores $\mathrm{D}_{2}$ dopaminérgicos, de onde o seu efeito negativo sobre a capacidade de exercício poderá ser explicado pela acção sobre a neurotransmissão dopaminérgica. Noutros estudos foram utilizadas a paroxetina [79, 
90] e a fluoxetina [68], inibidores da recaptação da serotonina. Studer et al. [79] e Wilson et al. [90], em dois estudos separados, verificaram um aumento da fadiga durante exercício prolongado, devido à administração de paroxetina $(20 \mathrm{mg})$. Meeusen et al. [68], porém, não observaram diferenças nas prestações de um contra-relógio (duração média de 90 min), em virtude da administração de fluoxetina. Este grupo já anteriormente reportara que administração de um inibidor da 5-HT (ritanserina) não tinha afectado a capacidade de realização em cicloergómetro de exercício de intensidade moderada $(65 \%$ do pico de potência) [67].

É possível que diferenças no nível de treino dos sujeitos que compõem as amostras possa, pelo menos em parte, explicar as conclusões diferentes encontradas a que chegaram os estudos anteriores. $\mathrm{O}$ treino pode ter como efeito reduzir a sensibilidade das vias da 5-HT e, assim, atenuar o efeito negativo sobre a capacidade de exercício do aumento da síntese e libertação de 5-HT. Esta assumpção é apoiada por resultados experimentais, os quais revelam que indivíduos treinados apresentam menor libertação de prolactina em resposta à buspirona, observação compatível com a diminuição do número ou da actividade dos receptores $5-\mathrm{HT}_{1 \mathrm{~A}}$ pós-sinápti$\cos [54,89]$. A hipótese do treino reduzir a sensibilidade serotoninérgica é, porém, contrariada por um outro estudo, no qual não se observaram diferenças entre indivíduos treinados e sedentários na resposta endócrina consequente à administração de d-fenfluramina, fármaco que aumenta libertação de 5-HT e inibe a sua recaptação neuronal [76]. Evidências experimentais mais recentes parecem também indicar que o treino físico é incapaz de produzir adaptações na actividade serotoninérgica cerebral [39]. Neste último estudo, verificou-se que um período de treino de 9 semanas ( $3 \times 30 \mathrm{~min}$ a $70 \%$ do $\mathrm{VO}_{2 \text { máx }}$ em ciclo-ergómetro, por semana) não produziu alteração na magnitude da resposta da prolactina a um teste de provocação com buspirona, apesar de ganhos substanciais na potência aeróbia máxima, em virtude do programa de treino aplicado [39]. A análise dos resultados individuais mostra, contudo, que em cerca de um quarto dos sujeitos da amostra foi evidente a atenuação da resposta endócrina após o treino [39], o que sugere que as adaptações nas vias da 5-HT dependem grandemente de variações inter-individuais. Permanece, ainda, o problema da selectividade parcial da buspirona, que impossibilita saber se as alterações da resposta hormonal são devidas à diminuição da sensibilidade das vias da 5HT ou ao aumento da actividade dopaminérgica [24]. Simultaneamente, continua a dúvida muito comum neste tipo de indagações: existindo uma menor sensibilidade dos receptores pós-sinápticos da 5-HT em atletas, será isso o produto do treino a que foram sujeitos ao longo de vários anos ou, antes, tratar-se-á de um fenómeno de selecção prévia proveniente da associação desta característica com o sucesso competitivo e a capacidade específica para o desempenho aeróbio.

Embora parte da investigação nesta área indique haver redução da tolerância ao exercício por indução farmacológica da actividade serotoninérgica cerebral, isto não implica, necessariamente, que em condições fisiológicas a fadiga seja originada pelo aumento da 5-HT. Nos estudos farmacológicos há a considerar a selectividade do fármaco, bem como os efeitos secundários. Por outro lado, há que atender que o resultado da acção do fármaco pode não ser compatível com as alterações fisiológicas. É difícil saber, em particular no homem, qual a concordância entre o aumento da quantidade de 5-HT cerebral resultante do exercício prolongado e aquele que é o resultado da acção dos fármacos.

\section{EFEITOS FÍSICOS E PSICOLÓGICOS DA INGESTÃO DE AMINOÁCIDOS DE CADEIA RAMIFICADA}

Durante o exercício há aumento da entrada de

AACR para o músculo, que é acompanhado de ligeira diminuição do teor destes aminoácidos no plasma sanguíneo. Valores medidos antes e após concluída uma maratona, revelam um decréscimo de $\sim 20 \%$ da concentração plasmática de AACR (de $470 \mu \mathrm{mol} / 1$ para $380 \mu \mathrm{mol} / \mathrm{l}$ ), enquanto que após 90 minutos de exercícios militares essa variação foi de 540 para 410 $\mu \mathrm{mol} / 1$ [17], correspondendo a uma diminuição da concentração plasmática de 20 a $25 \%$. Certos estudos laboratoriais mostram, igualmente, haver ligeira descida do teor plasmático dos AACR ao longo do exercício [78, 83]. Numa prova em cicloergómetro até à exaustão, realizada a uma intensidade de 70$75 \%$ da potência máxima, a concentração sanguínea 
dos AACR baixou de 442 para $380 \mu \mathrm{mol} / 1$ [83], enquanto Struder et al. [78] observaram uma descida de $25 \%$ da quantidade plasmática de AACR em tenistas após quatro horas de actividade.

Contudo, nem sempre é observada a descida da concentração plasmática de AACR em resultado do exercício. Numa prova de corrida, com uma duração de 60 minutos a $75 \%$ do $\mathrm{VO}_{2 \text { máx }}$, os teores plasmáticos de AACR não sofreram alteração, mesmo tendo os sujeitos iniciado a prova em jejum e com acentuada depleção do glicogénio muscular [94], sugerindo que esforços de intensidade moderada a elevada mas de duração não superior a uma hora, não alteram a concentração plasmática destes aminoácidos. No entanto, nem sempre existe diminuição do teor de AACR em circulação, em esforços com duração superior a uma hora $[49,59]$. Uma análise a 25 aminoácidos mostrou que a concentração plasmática de leucina não sofreu alteração após a realização de um ultra-triatlo, embora tenha havido diminuição da concentração sanguínea em 18 dos 25 aminoácidos doseados [59]. Graham et al. [49] verificaram, igualmente, que a concentração plasmática de AACR se manteve estável durante as três horas de realização de um exercício de extensão do joelho a $60 \%$ da potência máxima, tanto em indivíduos treinados como em sujeitos sedentários, embora neste caso haja a considerar a reduzida massa muscular envolvida no exercício.

Embora a descida da concentração plasmática de AACR associada ao exercício seja relativamente baixa, ou mesmo inexistente, particularmente em atletas de nível elevado de preparação, por redução da taxa de oxidação de leucina [65], ela associa-se ao aumento da concentração do TRP livre, decorrente da subida do teor de ácidos gordos livres em circulação [27, 94], resultando na descida do quociente TRP/AACR [17, 74, 77, 82, 94]. É possível, assim, que a ingestão de AACR, ao contrariar a subida do quociente TRP/AACR, previna ou retarde a fadiga central. Até à data, no entanto, os resultados da investigação não provam de forma categórica o efeito benéfico no rendimento físico da ingestão de AACR no período que antecede o exercício.

O efeito da ingestão de AACR no desempenho mental e físico foi avaliado em provas de corrida de 30 $\mathrm{km} \mathrm{e} 42.2 \mathrm{~km}$ (maratona) por Blomstrand et al.
[18]. Neste estudo, a ingestão de AACR elevou a sua concentração plasmática para valores superiores aos observados antes de iniciado o exercício e, em resultado, verificou-se melhoria no desempenho cognitivo após os $30 \mathrm{~km}$ de corrida. No que toca à prestação na prova de maratona, porém, não houve efeito significativo da ingestão de AACR, exceptuando num subgrupo de atletas, nos quais a ingestão de AACR melhorou os tempos de corrida. Em face destes resultados, os autores concluíram que a ingestão de AACR tem acção positiva no desempenho motor e cognitivo. Mais recentemente, Blomstrand et al. [22] verificaram que a ingestão de AACR melhorou a percepção subjectiva do esforço sem alterar, no entanto, o desempenho motor.

As conclusões de Blomstrand e colaboradores de que a ingestão de AACR contraria a fadiga central, com base nos resultados citados acima [1], tem motivado fortes críticas, dado que o estudo apresenta importantes falhas metodológicas, tais como ausência de controlo sobre os alimentos e líquidos ingeridos durante a prova pelos sujeitos da amostra e inexistência de um critério claro que tenha presidido à selecção do subgrupo da amostra no qual foi observado um efeito positivo da ingestão de AACR, levantando a suspeita de que se possa tratar de um resultado espúrio.

Diversos estudos mostram a inexistência de relação entre a concentração plasmática de AACR e a razão TRP livre/AACR e a percepção subjectiva de esforço [78] ou a fadiga durante exercício prolongado [62, 73, 74, 83]. van Hall et al. [83] avaliaram em atletas o efeito da ingestão de TRP ( $3.9 \mathrm{~g})$ e de AACR (7.8 g e $23.4 \mathrm{~g}$ ) na capacidade de exercício. Os resultados indicam que nenhum dos produtos ingeridos alterou a resistência à fadiga, quando o seu efeito foi comparado ao da ingestão de glucose. Estes resultados são semelhantes aos de um outro estudo, onde foram avaliados os efeitos da ingestão de glucose e de glucose mais AACR no desempenho de um contra-relógio de $100 \mathrm{~km}$ em cicloergómetro [62], e onde se verificou que os tempos gastos para completar os $100 \mathrm{~km}$ do contra-relógio foram semelhantes em todos os ensaios experimentais, independentemente da menor glicemia verificada na sessão placebo. Neste caso a ingestão de AACR traduziu-se na descida do valor do quociente TRP/AACR, mas sem que 
isso tenha tido efeito no resultado do contra-relógio. $\mathrm{O}$ facto da ingestão de glucose não ter, neste último estudo, melhorado o desempenho do contra-relógio, contrasta com aquilo que é aceite como acção da ingestão de glucose na prestação física em esforços de duração acima de 1 hora $[33,34]$. Na verdade, num estudo com um protocolo de exercício também de contra-relógio de $100 \mathrm{~km}$ em ciclo-ergómetro, a ingestão de glucose durante o exercício melhorou o desempenho [6]. A análise dos resultados individuais parece mostrar, no entanto, que o benefício da ingestão de glúcidos foi particularmente notório nos atletas com piores prestações [6].

É possível que o efeito da ingestão de AACR seja mais pronunciado quando o exercício é realizado em condições ambientais mais difíceis, particularmente em temperaturas ambientes elevadas. Mittleman et al. [69] verificaram que a ingestão de AACR melhorou o tempo até à exaustão em cerca de $14 \%$, num exercício em cicloergómetro a uma intensidade de $40 \% \mathrm{VO}_{2 \text { máx }}$, realizado sob uma temperatura ambiente de $\sim 34^{\circ} \mathrm{C}$, tendo este efeito sido observado em ambos os sexos. O interesse nestes resultados, porém, é condicionado pela baixa intensidade do exercício e pela baixa condição física dos sujeitos constituintes da amostra. Num estudo recente, a taxa de transferência de TRP entre a corrente sanguínea e o cérebro foi medida durante exercício no calor [72], tendo sido verificado não existir relação entre o aumento da percepção subjectiva de esforço e o balanço cerebral do TRP.

Outra proposta é que a ingestão de AACR é particularmente benéfica em exercício intervalado [77]. Esta hipótese foi testada, tendo-se chegado à conclusão que a ingestão de bebidas com glucose, numa concentração de $6 \%$ e $18 \%$, aumentou a resistência à fadiga, mas que a adição de AACR (7 g às soluções referidas) não trouxe efeitos adicionais [36], o que está de acordo com os resultados de outros estudos $[21,25]$.

Em resumo, os trabalhos laboratoriais, onde existe maior controlo sobre as variáveis em estudo, parecem demonstrar que a ingestão de AACR não tem efeito sobre a resistência à fadiga, contrariando as observações iniciais de Blomstrand et al. [19, 21]. Atendendo aos resultados dos estudos apresentados, a adição de AACR a soluções glucídicas não resulta num maior benefício, particularmente em atletas com nível elevado de treino. Por outro lado, a suplementação sistemática em aminoácidos pode trazer consequências que não são totalmente conhecidas. Por exemplo, Colombani et al. [31] registaram uma descida no teor plasmático total de aminoácidos durante uma corrida de maratona após um período de catorze dias de suplementação em aspartato de arginina. Perante estes resultados e depois de terem observado que o período de suplementação não trouxe nenhum benefício aparente nos parâmetros metabólicos durante a corrida, os autores concluíram haver motivo para uma reavaliação crítica quanto à prática de suplementação em aminoácidos como forma de melhorar o desempenho físico. Alguns dos potenciais efeitos da ingestão de AACR sobre o metabolismos muscular, serão seguidamente abordados.

\section{METABOLISMO MUSCULAR DOS AACR DURANTE O EXERCÍCIO}

Os AACR integram várias reacções metabólicas musculares, que em grande parte envolvem transferências do grupo amina entre a alanina, o glutamato e a glutamina e cuja velocidade aumenta em resposta à contracção muscular $[3,55]$. Durante o exercício aeróbio, com intensidade entre os 40 e $70 \%$ do $\mathrm{VO}_{2 \text { máx }}$, as concentrações intramusculares de glutamato e de alanina sofrem alterações importantes [48]. A concentração intramuscular do glutamato diminui em cerca de $50 \%$ nos primeiros 10 minutos da actividade muscular e mantém-se reduzida até à conclusão do exercício [88]. Este decréscimo rápido e acentuado da quantidade de glutamato existente no interior do músculo é devido, provavelmente, ao maior fluxo nas reaç̧ões que envolvem intermediários do ciclo de Krebs, e que aumentam a concentração destes últimos, num processo dito de anaplerose e que marca os momentos iniciais da contracção muscular [45].

Um das funções dos AACR é servirem de fonte para síntese de glutamato. A transferência do grupo amina dos AACR para o 2-oxoglutarato origina glutamato. Este, por sua vez, pode ser utilizado em duas reacções diferentes: 1 ) em reacção com o piruvato, sintetiza alanina e repõe o 2-oxoglutarato; 2) ao receber um grupo amina, forma glutamina [86, 
87]. Nesta segunda reacção, o glutamato faz o tamponamento da amónia, sendo esta a principal via de extracção da amónia do interior do músculo durante o exercício [86]. Assim, o glutamato desempenha um papel pivot nas reacções com aminoácidos. Serve para atenuar o acréscimo da quantidade intramuscular de amónia, e a sua combinação com o piruvato expande a pool dos intermediários do ciclo de Krebs [85]. É possível que esta última função do glutamato seja prioritária para suportar uma elevada taxa de síntese oxidativa de ATP, podendo ser a principal função dos aminoácidos durante o exercício [88]. As reacções mencionadas em cima explicam as variações nas concentrações sanguíneas e intramusculares dos aminoácidos envolvidos. Ao longo do exercício dá-se o efluxo muscular de glutamina e de alanina, em proporção com a intensidade e duração do exercício [50]. Simultaneamente, a taxa de entrada no músculo de glutamato e dos AACR aumenta. Assim, os AACR servem de fonte de glutamato, bem como de substrato energético [88]. Durante o exercício, o catabolismo proteico e a transaminação dos AACR parecem ser as principais fontes para o aumento do glutamato intramuscular, em particular nos indivíduos bem treinados [49].

A transaminação dos AACR resulta na formação dos derivados oxo-ácidos dos AACR, os quais podem ser posteriormente oxidados no músculo através da reacção irreversível catalizada pela enzima desidrogenase dos oxo-ácidos de cadeia ramificada. O exercício aumenta a actividade deste complexo enzimático [55], efeito que é potenciado por um maior aporte alimentar de proteínas e pela redução da quantidade do glicogénio muscular [84]. Esta activação enzimática resulta numa taxa acrescida de oxidação dos oxo-ácidos de cadeia ramificada, assim como numa taxa acrescida de transaminação dos AACR e formação de glutamato, já que estas duas reacções funcionam conjugadas.

\section{EVENTUAIS RISCOS DA INGESTÃO DE AACR}

Tem sido avançada a hipótese de que a maior taxa de oxidação dos AACR pode contribuir para a fadiga muscular durante o exercício prolongado, por aquela implicar diminuição da concentração de componentes do ciclo de Krebs e poder desencadear falência da síntese oxidativa de ATP $[75,86]$. Nos primeiros 5 minutos do exercício dá-se a expansão da pool de intermediários do ciclo de Krebs, com a reacção catalisada pela enzima alanina aminotransferase (piruvato + glutamato $\leftrightarrow 2$-oxoglutarato + alanina) a ser a principal razão desse aumento [75]. O 2-oxoglutarato serve de substrato na transaminação e oxidação dos AACR e se a esta utilização do 2-oxoglutarato acrescentar-se menor formação de piruvato, por decréscimo da taxa de glicólise associada à redução da quantidade de glicogénio muscular, poderá surgir uma redução crítica da taxa de síntese de ATP [84, 86]. Esta hipótese é sustentada, em grande parte, por dados obtidos em indivíduos portadores da doença de McArdle [86], caracterizada por ausência da enzima miofosforilase e incapacidade de utilização do glicogénio muscular no metabolismo energético. Nestes sujeitos, a ingestão de AACR (10 g de leucina, isoleucina e valina) 30 minutos antes de iniciado o exercício aumentou a concentração plasmática de amónia, a frequência cardíaca e a percepção subjectiva de esforço, tendo ainda acelerado a ocorrência de fadiga [86]. Uma maior produção de amónia durante exercício e em resultado da ingestão de amónia, foi igualmente observada em sujeitos sem patologia muscular [58, 60, 61, 83].

A amónia é potencialmente tóxica, actuando no músculo ou no sistema nervoso central, onde pode bloquear a neurotransmissão ou reduzir a síntese de neurotransmissores [12]. No entanto, e como vimos em pontos anteriores, não existe indicação de ocorrência de alterações tóxicas secundárias à ingestão de AACR. Contudo, não existem dados exaustivos sobre o efeito dos AACR em condições muito particulares, como são os casos do exercício em altitude ou com temperatura ambiente elevada.

$\mathrm{O}$ outro mecanismo de fadiga associada à ingestão de AACR é, como já foi salientado, a possível redução na concentração de compostos do ciclo de Krebs, que nos sujeitos com doença de McArdle seria agravada pela taxa reduzida de glicólise e de formação de piruvato. Situação semelhante poderá ocorrer em qualquer indivíduo com o aumento da duração do exercício e com o esgotamento das reservas de glicogénio muscular. Contudo, os resultados experimentais não confirmam que exista relação estreita entre a quantidade de glicogénio muscular e a concentração dos intermediários do ciclo de Krebs [11, 47]. 
Por outro lado, não é clara a importância da concentração dos intermediários do ciclo de Krebs e a taxa de síntese oxidativa de ATP. Contrariamente à hipótese, as observações experimentais indicam que uma menor quantidade de glicogénio muscular potencia o fenómeno de anaplerose observado nos instantes iniciais do exercício [11, 47]. Uma relação estreita entre a concentração dos intermediários do ciclo de Krebs e o fluxo de reacções na via oxidativa parece também não existir, uma vez que ao longo de hora e meia de exercício houve decréscimo progressivo na concentração dos intermediários do ciclo de Krebs, mas sem alteração do consumo muscular de $\mathrm{O}_{2}$ [46]. Estas evidências experimentais, conjuntamente com a reduzida capacidade muscular de oxidação dos AACR, tornam pouco provável em sujeitos saudáveis a hipótese de fadiga muscular originada pela ingestão de AACR.

\section{CONCLUSÃO}

Durante o exercício prolongado poderá haver acréscimo na síntese cerebral de serotonina devido ao aumento da fracção livre do TRP em circulação no plasma sanguíneo, associado a uma diminuição eventual na concentração plasmática dos AACR. A ingestão de AACR poderá corrigir as alterações metabólicas que aumentam a entrada de TRP para o cérebro, daí poder ser útil na prevenção da fadiga central. Uma grande parte dos estudos realizados, porém, refuta que os AACR possuam uma acção ergogénica. Por outro lado, a ingestão de AACR aumenta a produção de amónia, o que tem efeitos potenciais negativos.

Em resumo, parece não haver justificação para introduzir a ingestão de AACR, antes e durante o exercício, como estratégia para melhorar o desempenho. Contudo, a ingestão de aminoácidos, em particular de AACR, pode trazer benefícios de outra natureza, tais como a redução do catabolismo proteico durante o esforço, como é sugerido por De Palo et al. [37] ou durante a recuperação [23] ou, ainda, o abrandamento do efeito de imunosupressão associado a estados de fadiga pós-esforço elevada [13], que não foram abordados neste artigo.

\section{CORRESPONDÊNCIA}

Paulo A.S. Armada da Silva

Faculdade de Motricidade Humana

Universidade Técnica de Lisboa

Estrada da Costa da Costa

Cruz-Quebrada, Portugal

parmada@fmh.utl.pt 


\section{BIBLIOGRAFIA}

1. Abbate F, De Ruiter C J, Offringa C, Sargeant A J, De Haan A (2002). In situ rat fast skeletal muscle is more efficient at submaximal than at maximal activation levels. J Appl Physiol 92: 2089-96.

2. Acworth I, Nicholass J, Morgan B, Newsholme E A (1986). Effect of sustained exercise on concentrations of plasma aromatic and branched-chain amino acids and brain amines. Biochem Biophys Res Commun 137: 149-53.

3. Aftring R P, Block K P, Buse M G (1986). Leucine and isoleucine activate skeletal muscle branched-chain alpha-keto acid dehydrogenase in vivo. Am J Physiol 250: E599-604.

4. Ahlenius S, Kaur P, Salmi P (1997). Biphasic effects of 8$\mathrm{OH}-\mathrm{DPAT}$ on endurance of treadmill performance in the male rat. Eur Neuropsychopharmacol 7: 89-94.

5. Anderson I M, Parry-Billings M, Newsholme E A, Fairburn C G, Cowen P J (1990). Dieting reduces plasma tryptophan and alters brain 5-HT function in women. Psychol Med 20: 785-91.

6. Angus D J, Hargreaves M, Dancey J, Febbraio M A (2000). Effect of carbohydrate or carbohydrate plus medium-chain triglyceride ingestion on cycling time trial performance. $J$ Appl Physiol 88: 113-9.

7. Azmitia E C,Gannon P J (1986). The primate serotonergic system: a review of human and animal studies and a report on Macaca fascicularis. Adv Neurol 43: 407-68.

8. Bailey S P, Davis J M, Ahlborn E N (1992). Effect of increased brain serotonergic activity on endurance performance in the rat. Acta Physiol Scand 145: 75-6.

9. Bailey S P, Davis J M, Ahlborn E N (1993). Serotonergic agonists and antagonists affect endurance performance in the rat [see comments]. Int J Sports Med 14: 330-3.

10. Bailey S P, Davis J M, Ahlborn E N (1993).

Neuroendocrine and substrate responses to altered brain 5-HT activity during prolonged exercise to fatigue. J Appl Physiol 74: 3006-12.

11. Baldwin J, Snow R J, Gibala M J, Garnham A, Howarth K, Febbraio M A (2003). Glycogen availability does not affect the TCA cycle or TAN pools during prolonged, fatiguing exercise. J Appl Physiol 94: 2181-7.

12. Banister E W, Cameron B J (1990). Exercise-induced hyperammonemia: peripheral and central effects. Int $J$ Sports Med 11 Suppl 2: S129-42.

13. Bassit R A, Sawada L A, Bacurau R F, Navarro F, Martins E, Jr., Santos R V, Caperuto E C, Rogeri P, Costa Rosa L F (2002). Branched-chain amino acid supplementation and the immune response of long-distance athletes. Nutrition 18: 376-9.

14. Becquet D, Faudon M, Hery F (1990). The role of serotonin release and autoreceptors in the dorsalis raphe nucleus in the control of serotonin release in the cat caudate nucleus. Neuroscience 39: 639-47.

15. Bergstrom J, Hermansen L, Hultman E, Saltin B (1967). Diet, muscle glycogen and physical performance. Acta Physiol Scand 71: 140-50.

16. Bergstrom J,Hultman E (1967). Synthesis of muscle glycogen in man after glucose and fructose infusion. Acta Med Scand 182: 93-107.

17. Blomstrand E, Celsing F, Newsholme E A (1988). Changes in plasma concentrations of aromatic and branched-chain amino acids during sustained exercise in man and their possible role in fatigue. Acta Physiol Scand 133: 115-21.

18. Blomstrand E, Hassmen P, Ekblom B, Newsholme E A (1991). Administration of branched-chain amino acids during sustained exercise- effects on performance and on plasma concentration of some amino acids. Eur J Appl Physiol 63: 83-8.

19. Blomstrand E, Hassmen P, Newsholme E A (1991). Effect of branched-chain amino acid supplementation on mental performance. Acta Physiol Scand 143: 225-6.

20. Blomstrand E,Newsholme E A (1992). Effect of branchedchain amino acid supplementation on the exercise- induced change in aromatic amino acid concentration in human muscle. Acta Physiol Scand 146: 293-8.

21. Blomstrand E, Andersson S, Hassmen P, Ekblom B, Newsholme E A (1995). Effect of branched-chain amino acid and carbohydrate supplementation on the exerciseinduced change in plasma and muscle concentration of amino acids in human subjects. Acta Physiol Scand 153: 8796.

22. Blomstrand E, Hassmen P, Ek S, Ekblom B, Newsholme E A (1997). Influence of ingesting a solution of branchedchain amino acids on perceived exertion during exercise. Acta Physiol Scand 159: 41-9.

23. Blomstrand E, Saltin B (2001). BCAA intake affects protein metabolism in muscle after but not during exercise in humans. Am J Physiol Endocrinol Metab 281: E365-74.

24. Bridge M W, Marvin G, Thompson C E, Sharma A, Jones D A, Kendall M J (2001). Quantifying the 5-HT1A agonist action of buspirone in man. Psychopharmacology (Berl) 158: 224-9.

25. Calders P, Matthys D, Derave W, Pannier J L (1999). Effect of branched-chain amino acids (BCAA), glucose, and glucose plus BCAA on endurance performance in rats. Med Sci Sports Exerc 31: 583-7.

26. Castell L M, Yamamoto T, Phoenix J, Newsholme E A (1999). The role of tryptophan in fatigue in different conditions of stress. Adv Exp Med Biol 467: 697-704.

27. Chaouloff F, Kennett G A, Serrurrier B, Merino D, Curzon G (1986). Amino acid analysis demonstrates that increased plasma free tryptophan causes the increase of brain tryptophan during exercise in the rat. J Neurochem 46: 1647-50.

28. Chaouloff F, Laude D, Elghozi J L (1989). Physical exercise: evidence for differential consequences of tryptophan on 5 -HT synthesis and metabolism in central serotonergic cell bodies and terminals. J Neural Transm 78: 121-30.

29. Chaouloff F (1993). Physiopharmacological interactions between stress hormones and central serotonergic systems. Brain Res Brain Res Rev 18: 1-32.

30. Chaouloff F (1997). Effects of acute physical exercise on central serotonergic systems. Med Sci Sports Exerc 29: 5862 .

31. Colombani P C, Bitzi R, Frey-Rindova P, Frey W, Arnold M, Langhans W, Wenk C (1999). Chronic arginine aspartate supplementation in runners reduces total plasma amino acid level at rest and during a marathon run. Eur J Nutr 38: 263-70.

32. Costill D L, Dalsky G P, Fink W J (1978). Effects of caffeine ingestion on metabolism and exercise performance. Med Sci Sports 10: 155-8.

33. Coyle E F, Hagberg J M, Hurley B F, Martin W H, Ehsani A A, Holloszy J O (1983). Carbohydrate feeding during pro- 
longed strenuous exercise can delay fatigue. J Appl Physiol 55: 230-5.

34. Coyle E F,Coggan A R (1984). Effectiveness of carbohydrate feeding in delaying fatigue during prolonged exercise. Sports Med 1: 446-58.

35. Cunningham V J, Hay L, Stoner H B (1975). The binding of L-tryptophan to serum albumins in the presence of nonesterified fatty acids. Biochem J 146: 653-8.

36. Davis J M, Welsh R S, De Volve K L, Alderson N A (1999). Effects of branched-chain amino acids and carbohydrate on fatigue during intermittent, high-intensity running. Int $J$ Sports Med 20: 309-14.

37. De Palo E F, Gatti R, Cappellin E, Schiraldi C, De Palo C B, Spinella P (2001). Plasma lactate, GH and GH-binding protein levels in exercise following BCAA supplementation in athletes. Amino Acids 20: 1-11.

38. Delgado P L, Charney D S, Price L H, Aghajanian G K, Landis H, Heninger G R (1990). Serotonin function and the mechanism of antidepressant action. Reversal of antidepressant-induced remission by rapid depletion of plasma tryptophan [see comments]. Arch Gen Psychiatry 47: 411-8.

39. Dwyer D, Flynn J (2002). Short term aerobic exercise training in young males does not alter sensitivity to a central serotonin agonist. Exp Physiol 87: 83-9.

40. Emori T, Sugiyama K, Nagase S (1983). Tryptophan metabolism in analbuminemic rats. J Biochem (Tokyo) 94: 62332.

41. Farris J W, Hinchcliff K W, McKeever K H, Lamb D R, Thompson D L (1998). Effect of tryptophan and of glucose on exercise capacity of horses. J Appl Physiol 85: 807-16.

42. Fitts R H, Courtright J B, Kim D H, Witzmann F A (1982). Muscle fatigue with prolonged exercise: contractile and biochemical alterations. Am J Physiol 242: C65-73.

43. Fitts R H (1994). Cellular mechanisms of muscle fatigue. Physiol Rev 74: 49-94.

44. Ftaiti F, Grelot L, Coudreuse J M, Nicol C (2001). Combined effect of heat stress, dehydration and exercise on neuromuscular function in humans. Eur J Appl Physiol 84: 87-94.

45. Gibala M, MacLean D, Graham T, Saltin B (1997). Anaplerotic processes in human skeletal muscle during brief dynamic exercise. J Physiol (Lond) 502: 703-713.

46. Gibala M J, Gonzalez-Alonso J, Saltin B (2002). Dissociation between muscle tricarboxylic acid cycle pool size and aerobic energy provision during prolonged exercise in humans. J Physiol 545: 705-13.

47. Gibala M J, Peirce N, Constantin-Teodosiu D, Greenhaff $P$ L (2002). Exercise with low muscle glycogen augments TCA cycle anaplerosis but impairs oxidative energy provision in humans. J Physiol 540: 1079-86.

48. Graham T E,MacLean D A (1992). Ammonia and amino acid metabolism in human skeletal muscle during exercise. Can J Physiol Pharmacol 70: 132-41.

49. Graham T E, Turcotte L P, Kiens B, Richter E A (1995). Training and muscle ammonia and amino acid metabolism in humans during prolonged exercise. J Appl Physiol 78: 725-35.

50. Graham T E,MacLean D A (1998). Ammonia and amino acid metabolism in skeletal muscle: human, rodent and canine models. Med Sci Sports Exerc 30: 34-46.

51. Hermansen L, Hultman E, Saltin B (1967). Muscle glycogen during prolonged severe exercise. Acta Physiol Scand
71: 129-39.

52. Hillegaart V, Wadenberg M L, Ahlenius S (1989). Effects of 8-OH-DPAT on motor activity in the rat. Pharmacol Biochem Behav 32: 797-800.

53. Jacobs B L,Azmitia E C (1992). Structure and function of the brain serotonin system. Physiol Rev 72: 165-229.

54. Jakeman P M, Hawthorne J E, Maxwell S R, Kendall M J, Holder G (1994). Evidence for downregulation of hypothalamic 5-hydroxytryptamine receptor function in endurance-trained athletes. Exp Physiol 79: 461-4.

55. Kasperek G J (1989). Regulation of branched-chain 2-oxo acid dehydrogenase activity during exercise. Am J Physiol Endocrinol Metab 256: E186-190.

56. Kay D, Marino F E, Cannon J, St Clair Gibson A, Lambert M I, Noakes T D (2001). Evidence for neuromuscular fatigue during high-intensity cycling in warm, humid conditions. Eur J Appl Physiol 84: 115-21.

57. Knowles R G,Pogson C I (1984). Characteristics of tryptophan accumulation by isolated rat forebrain synaptosomes. J Neurochem 42: 663-9.

58. Kreider R B, Miriel V, Bertun E (1993). Amino acid supplementation and exercise performance. Analysis of the proposed ergogenic value. Sports Med 16: 190-209.

59. Lehmann M, Huonker M, Dimeo F, Heinz N, Gastmann U, Treis N, Steinacker J M, Keul J, Kajewski R, Haussinger D (1995). Serum amino acid concentrations in nine athletes before and after the 1993 Colmar ultra triathlon. Int $J$ Sports Med 16: 155-9.

60. MacLean D A,Graham T E (1993). Branched-chain amino acid supplementation augments plasma ammonia responses during exercise in humans. J Appl Physiol 74: 2711-7.

61. MacLean D A, Graham T E, Saltin B (1996). Stimulation of muscle ammonia production during exercise following branched-chain amino acid supplementation in humans. $J$ Physiol 493: 909-22.

62. Madsen K, MacLean D A, Kiens B, Christensen D (1996). Effects of glucose, glucose plus branched-chain amino acids, or placebo on bike performance over $100 \mathrm{~km}$. J Appl Physiol 81: 2644-50.

63. Marsden C A, Conti J, Strope E, Curzon G, Adams R N (1979). Monitoring 5-hydroxytryptamine release in the brain of the freely moving unanaesthetized rat using in vivo voltammetry. Brain Res 171: 85-99.

64. Marvin G, Sharma A, Aston W, Field C, Kendall M J, Jones D A (1997). The effects of buspirone on perceived exertion and time to fatigue in man. Exp Physiol 82: 1057-60.

65. McKenzie S, Phillips S M, Carter S L, Lowther S, Gibala M J, Tarnopolsky M A (2000). Endurance exercise training attenuates leucine oxidation and BCOAD activation during exercise in humans. Am J Physiol Endocrinol Metab 278: E580-7.

66. Meeusen R, Thorre K, Chaouloff F, Sarre S, De Meirleir K, Ebinger G, Michotte Y (1996). Effects of tryptophan and/or acute running on extracellular 5-HT and 5- HIAA levels in the hippocampus of food-deprived rats. Brain Res 740: 245-52.

67. Meeusen R, Roeykens J, Magnus L, Keizer H, De Meirleir $\mathrm{K}$ (1997). Endurance performance in humans: the effect of a dopamine precursor or a specific serotonin $(5-\mathrm{HT} 2 \mathrm{~A} / 2 \mathrm{C})$ antagonist. Int J Sports Med 18: 571-7.

68. Meeusen R, Piacentini M F, Van Den Eynde S, Magnus L, De Meirleir K (2001). Exercise performance is not influenced by 
a 5-HT reuptake inhibitor. Int J Sports Med 22: 329-36.

69. Mittleman K D, Ricci M R, Bailey S P (1998). Branchedchain amino acids prolong exercise during heat stress in men and women. Med Sci Sports Exerc 30: 83-91.

70. Newsholme E A, Acworth I N, Blomstrand E (1987). Amino acids, brain neurotransmitters and a functional link between muscle and brain that is important in sustained exercise. In G. Benzi (Ed.) Advances in Myochemistry. London: John Libbey Eurotext Ltd, 127-133.

71. Newsholme E A, Blomstrand E, Ekblom B (1992). Physical and mental fatigue: metabolic mechanisms and importance of plasma amino acids. Br Med Bull 48: 477-95.

72. Nybo L, Nielsen B, Blomstrand E, Moller K, Secher N (2003). Neurohumoral responses during prolonged exercise in humans. J Appl Physiol 95: 1125-31.

73. Pitsiladis Y P,Maughan R J (1999). The effects of exercise and diet manipulation on the capacity to perform prolonged exercise in the heat and in the cold in trained humans. J Physiol 517: 919-30.

74. Pitsiladis Y P, Strachan A T, Davidson I, Maughan R J (2002). Hyperprolactinaemia during prolonged exercise in the heat: evidence for a centrally mediated component of fatigue in trained cyclists. Exp Physiol 87: 215-26.

75. Sahlin K, Katz A, Broberg S (1990). Tricarboxylic acid cycle intermediates in human muscle during prolonged exercise. Am J Physiol 259: C834-41.

76. Strachan A T, Maughan R J (1999). The hormonal response to a d-fenfluramine challenge in trained and sedentary men. Med Sci Sports Exerc 31: 547-53.

77. Struder H K, Hollmann W, Duperly J, Weber K (1995). Amino acid metabolism in tennis and its possible influence on the neuroendocrine system. Br J Sports Med 29: 28-30.

78. Struder H K, Hollmann W, Platen P, Duperly J, Fischer H G, Weber K (1996). Alterations in plasma free tryptophan and large neutral amino acids do not affect perceived exertion and prolactin during $90 \mathrm{~min}$ of treadmill exercise. Int J Sports Med 17: 73-9.

79. Struder H K, Hollmann W, Platen P, Donike M, Gotzmann A, Weber K (1998). Influence of paroxetine, branchedchain amino acids and tyrosine on neuroendocrine system responses and fatigue in humans. Horm Metab Res 30: 188194.

80. Struder H K,Weicker H (2001). Physiology and pathophysiology of the serotonergic system and its implications on mental and physical performance. Part II. Int J Sports Med 22: 482-97.

81. Struder H K,Weicker H (2001). Physiology and pathophysiology of the serotonergic system and its implications on mental and physical performance. Part I. Int J Sports Med 22: $467-81$.

82. Tanaka H, West K A, Duncan G E, Bassett D R, Jr. (1997). Changes in plasma tryptophan/branched chain amino acid ratio in responses to training volume variation. Int $J$ Sports Med 18: 270-5.

83. van Hall G, Raaymakers J S, Saris W H, Wagenmakers A J (1995). Ingestion of branched-chain amino acids and tryptophan during sustained exercise in man: failure to affect performance. J Physiol (Lond) 486: 789-94.

84. van Hall G, MacLean D A, Saltin B, Wagenmakers A J (1996). Mechanisms of activation of muscle branchedchain alpha-keto acid dehydrogenase during exercise in man. J Physiol 494: 899-905.
85. Van Hall G, Saltin B, Wagenmakers A J (1999). Muscle protein degradation and amino acid metabolism during prolonged knee-extensor exercise in humans. Clin Sci (Colch) 97: 557-67.

86. Wagenmakers A J, Coakley J H, Edwards R H (1990). Metabolism of branched-chain amino acids and ammonia during exercise: clues from McArdle's disease. Int J Sports Med 11 Suppl 2: S101-13.

87. Wagenmakers A J (1992). Amino acid metabolism, muscular fatigue and muscle wasting. Speculations on adaptations at high altitude. Int J Sports Med 13 Suppl 1: S110-3.

88. Wagenmakers A J (1998). Muscle amino acid metabolism at rest and during exercise: role in human physiology and metabolism. Exerc Sport Sci Rev 26: 287-314.

89. Weicker H,Struder H K (2001). Influence of exercise on serotonergic neuromodulation in the brain. Amino Acids 20: 35-47.

90. Wilson W M,Maughan R J (1992). Evidence for a possible role of 5-hydroxytryptamine in the genesis of fatigue in man: administration of paroxetine, a 5-HT re-uptake inhibitor, reduces the capacity to perform prolonged exercise. Exp Physiol 77: 921-4.

91. Yamamoto T, Castell L M, Botella J, Powell H, Hall G M, Young A, Newsholme E A (1997). Changes in the albumin binding of tryptophan during postoperative recovery: a possible link with central fatigue? [published erratum appears in Brain Res Bull 1997;44(6):735]. Brain Res Bull 43: 43-6.

92. Yamamoto T,Newsholme E A (2000). Diminished central fatigue by inhibition of the L-system transporter for the uptake of tryptophan. Brain Res Bull 52: 35-8.

93. Young S N,Gauthier S (1981). Tryptophan availability and the control of 5-hydroxytryptamine and tryptamine synthesis in human CNS. Adv Exp Med Biol 133: 221-30.

94. Zanker C L, Swaine I L, Castell L M, Newsholme E A (1997). Responses of plasma glutamine, free tryptophan and branched-chain amino acids to prolonged exercise after a regime designed to reduce muscle glycogen. Eur $J$ Appl Physiol 75: 543-8. 